sultaron de gran apoyo para el desarrollo de la autonomía politica y administrativa a traves de varias normativas como la refoma de la Constitución del 2008, COOTAD 2010 entre otras que controlan las distintas competencias con el objetivo de generar lado, el modelo uruguayo no corrió con la misma suerte por la poca importancia que se dio a la implementación de esta institucionalidad que tenía como objetivo el fortalecimiento de la autonomía municipal.

El sistema ecuatoriano se ajusta a los modelos anglosajón y al europeo puesto que cuenta con una normativa que brinda los gobiernos locales una autonomia politica, financiera y adapunta al fortalecimiento del sistema electoral y de la particapunta al fortalecimiento del sistema electoral y de la partic-
pación ciudadana, mediante un sistema electoral proporciona y varios mecanismos que permiten controlar la administración de la gobernanza local. Mientras que el sistema uruguayo se inclina más al modelo anglosajón por la falta de interés de participación de la ciudadanía en elecciones electorales municipales lo que origina varios problemas de interés socio-teritorial origimejorar el desempleo en la prestación de senvicis.

A pesar de la gran diferencia de los modelos de Ecuador y Uruguay, la gran ambición es lograr que los gobiernos locales lleguen a cumplir las necesidades de la población además de brindar eficiencia en los servicios que ayuden a alcanzar el denominado Buen Vivir de la ciudadanía.

\section{Referencias}

ESIC. (2015). Gobierno Abierto Proceso y resultados de Uruguay. Uruguay: Agencia de gobierno electrónico y sociedad de información

Altair, J. (2010). Descentralización municipal en Uruguay estreno de un nivel de gobierno que no estusiasmó a la

Revista I Iervamericana de Estudios Municipales, 83-110.
Alvarez, S. (2015). El proceseso de descentralizacion en América ina y Ecuador. Ecuador: Movimiento Alianza PAIS.

Bedón, G., \& Guerra, G. (2012). Nuevo modelo de descentralización en el Ecuador a partir de la Constitución del 2008. XV Congreso Internacional del CLAD sobre la Reforma del Estado y de la
Administración Pública, (págs. 1-19). Colombia. Consejo Nacional de Competencias: http://murw competenciss. gob.ecl

Congreso de Intendentes. (18 de Junio de 2013). Municipios. Obtenido de Congreso de Intendentes de Uruguay: http://www.cigub. uy/index.php/2013-06-18-12-01-34/2013-06-18-12-03-42

2014. Consejo Nacional Electoral. (2014). Elecciones Seccionales es/estadisticas/bases-de-datos/category/346-elecciones-seccion-

Consejo Nacional Electoral. (Abril de 2017). Resultados Prehttps://resultados2017-2. cne gob.ec/frmResultados. aspx Consitucion de la Republica del Ecuador. (2008). Consitución de la República del Ecuador. Ecuador.

Constitución de la Republica Oriental de Uruguay. (1967). Constitución de la Republica Oriental de Uruguay. Uruguay.
COOTAD. (2010). Código Orgánico de Organización Territorial, Autonomía y Descentralización. Ecuador: Ministerio de la Coordinación .

2014). Elecciones 2014 Segunda Elección Oruguay. (NoviemElectoral de la República Oriental del Uruguay: http://segundeleccion.

Corte Electoral de la República Oriental del Uruguay. (Mayo de 2015). Elecciones Departamentales Municipales 2015. Obtenido de Corte Electoral de la República Oriental del Uruguay: http://elecciones-

Decreto No 460/016. (2016). APROBACION DEL “3ER. PLAN DE ACCION NACIONAL DE GOBIERNO ABIERTO DE URUGUAY 2016 8". Uruguay: IMPO centro de información ofici

Decreto No 33.209. (2009). Decreto № 33.209 de la Junta (106 de ág. 3.

(2014). Uruguay en cifras 2014. Uruguay: Instituto Naciona

INEC. (2010). Ecuador en cifras. Obtenido de Instituto Naciona de Estadistica y Censos: http://www.ecuadorencifras.gob.ec/proyecpoblacionales/

Larenas, R., Varela, C., Quiro, L., Narváez, M., \& Sáenz, M. tralizad: Un Estado del Ate de la Descen a la concentración descenuador: CONGOPE.

Ley 19.272. (2014). Descentralización Pollitica y Participación adana. Uruguay.

Ley Orgánica Electoral, Código de la Democracia. (2009). ConMunicipales. Ecuador: Asamblea Nacional

Miralles, E., Calabre, L., \& Weber, R. (2010). VII Campus Euroamericano de Cooperación Cultural - Descentralización Europa y Amenica Latina. España. Organización de Es Navaro, la Clencia y la Cullura.

(2003). The structures of and cultural factors. En Local reaction to Globalization Processes. New York: F. Entrena.

Nickson, A. (2011). Where Is Local Government Going in Latin a? A Comparative Perspective. Reino Unido: ICLD.

Parlamento de la Repubilica Oriental del Uruguay. (2016). Paramento del Uruguay. Obtenido de hitps://parlamento.gub. uy: https:/

Ramírez, M., \& Navarro, C. (2002). Enfoques para el estudio del municipal. Redalyc, 11-26.

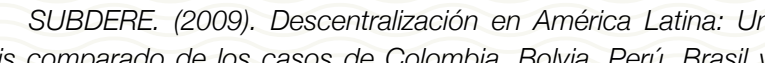
Argentina. Chile: Subsecretaría de Desarrollo Regional y Administrativo de Chile.

Tarrow, S. (1977). Entre el centro y la periferia. New Haven: Yale

Weber, M. (1968). Economía y sociedad: un contorno de sociologia interpretativa. Nueva York: Prensa de Bedminster.

\title{
Metodología Prospectiva para el estudio de la Seguridad Social
}

Frank Rivas - Torres'

Fecha de recepción: 5 de Septiembre 2017

Fecha de aceptación: 29 de Septiembre 2017

Resumen

lobjetivo de este trabajo es explicar una metodología cualitativa apoyada en los mapas conceptuales y/o conceptos estrucurados que utilizan grupos de personas expertas en un tema de Impacto Cruzado (MICMAC), a través de la cual puede hacerse un análisis prospectivo de la seguridad social. Por lo que se exponen aspectos detallados las variables denominadas claves que se obtienen, a través del análisis bibliográfico, apoyado en un discurso descriplivo. Finalmente, se refiere istado de variable curso del artículo, el cual es producto de la técnica suscrita.

\section{Palabras clave:}

Abstract

The objective of this paper is to explain a qualitative methodology supported by conceptual maps and $\%$ or structured concepts used by groups of experts in a subject or with a direct relation-
ship of the problem under study and the Cross Impact Matrix ship of the problem under study and the Cross Impact Matrix (MICMAC), hrough from which a prospeclive social security through bibliographic analysis supported by a descriptive discourse. Finally, it refers to a list of variables product of the exercise explained in the course of the article, which is a product of the subscribed technique.

Keywords: Social security, structural analysis, prospective

Introducción

sespiritu proteccionista de la de la seguridad social y su reforma bien sea de tipo estructural y/o institucional, deyitivamente es un fenómeno que deviene de la globalización, yes sensible a las instituciones elementales del Estado venezolano. Todavia no está nada claro la relación que existe enalgunos datos que indican al menos tres tendencio, se tienen concepción organizacional típica de la era industrial (ho la fijo, especialización fábrica tiempo libre) se ha alterado; 2) que en esta época se abren mútiples opciones para el trabajo remunerado; 3) que, de ser ciertas estas opciones, se derivan algunas consecuencias para la estabilidad politica en la región. (Rivas T., Frank E. y Silva D., María F., 2017).

Ahora bien, la Seguridad Social ha enraizado su objeto de estudio como ciencia autónoma, por lo tanto, está reclamando diversas metodologlas que den cuenta de conclusiones plausibles para el aporte de teoria en el area. En este sentido, las la seguridad social y los nuevos sistemas de protección al trabajador y al ciudadano en general, requieren inc dos alternativos de las ciencias sociales, tal como es el caso del análisis del discurso sobre la seguridad social, o la opinion tesce le perspectiva documental y cuallitativa de expertos en el . acto Cruzado, (MICMAC) de la obra Caja de herramientes de Michel Godet, además la técnica del mapeo o mapa conceptual de M. K. Trochim (1989).

conceptual o mapeo hace referencia a conceptos estructurados que utilizan grupos de personas expertas en un tema o con una relación directa del problema bajo estudio, en este caso la seguridad social. Esta noción permite desarrollar un marco conceptual que puede dirigir la evaluación mando cior s variables que permitan analizar de maneras objetiva una situación dada. En el caso de este trabajo, se pretende utilizar este tipo de metodología dado que el interés es estudiar por el Estado venezolano, y específicamente el caso de la sepuldad social que en los ultimos años ha sido punto do evaluación ara convertiro en un sistema eficiente, con autonomía, que

\section{etodologia}

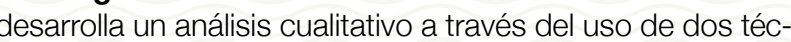
conceptuales de Trochim (1989) y la prospectiva, que permiten la generación de variables clave en dos sesiones ar un conversatorio, por parte de un grupo de interés focalizade la comunidad o representantes de grupos de la gerencia administrativa, pública o privada; académicos o legisladores); cesadas por un software sistema de seguridad social son proráfica de las variables mas importantes para el sistema de seguridad social, y su influencia sobre el sistema. Por tanto, el nvestigador bajo este escenario desarrolla un análisis sobre las prioridades a la hora de la implementación de la seguridad

\section{Resultados}

obtuvieron 25 variables claves que permitieron realizar un ercicio de hermenéutica intelectual a través de la representación grafica donde se destacan cuáles son las más importantes para la implementación del sistema de Seguridad social. Las que cualquier influencia ejercida sobre una de ellas afecta a toas las demás y viceversa. Este método presenta la ventaja de ermitir un estudio cualitativo del sistema en el cual se sustenta bilaciones. Sobre la Metodología de Guillermo Trochim
Se estimó conveniente articular la técnica de los grupos de dis- 
cusión -conversatorio-, y el diseño de mapas conceptuales
utilizados en la planificación y evaluación de Guillermo Trochim (1989), la cual cuenta con su propia metodología y sistematicidad para la interpretación cuallaliva del objeto de estudio. parámetros:

1. El estudio de las diversas posturas y perspectivas que adopten los actores porque forman parte de la realidad que se estudia. Esto implica considerar la forma en que los representantes de la seguridad social se relacionan con la sociedad. 2. El estudio enmarcado en la seguridad social implica por mismo analizar la intervención de los actores en término de recoger
sus percepciones -actitudes-actuaciones-disposiciones entre otros aspectos - lo que exige analizar y conocer la intensidad y
profundidad entre lo que viene a ser el vinculo de lo que se tiene normado-creado y lo que se hace.

Por ello Callejo (2001), hace énfasis en la técnica/métodos que se emplean en los grupos de discusión, como un ejercicio para equipo se ha concretado en diversas prácticas que, por sus objetivos y desarrollo pretende establecer vinculación con diversos grupos de trabajo; se habla por ejemplo de entrevistas de grupo, grupo focalizado o enfocado (focus group), grupos nominales, grupos de expertos, grupos delphi, grupos enfren-
tados y el diseño de mapas conceptuales utilizados en la planifitados y el diseño de mapas conceptuales utilizados en la planif cación y evaluación, entre otros. Por tanto, esta investigación
diseñó la reunión de grupos de expertos a través de un conversatorio, lo cual permitirá obtener insumos valiosos - valor agregado - acerca del tema desarrollado.

Por tanto, de acuerdo con este arquetipo de técnica, la verdad la praxis cientffica, y también del problema in fundamental en en constatar o reproducir estos hechos con el fin de estabnta vinculaciones que permitan obtener respuestas a los problemas planteados.

El diseño de mapas conceptuales y la Matriz de Impacto Cruzado aplicados al sist ma de Segundad social

En este contexto, el mapa conceptual es una representación ideas del mismo relacionadas con el tue muestra todas trabajando; asimismo, esta representación muestra como estas ideas están relacionadas entre sí y, si es necesario, puede mostrar cuales ideas son más relevantes, importantes o pertinentes. En este sentido, se explicará la técnica del mapeo

El diseño de un mapa conceptual constituye un incentivo para seguir desarrollando un trabajo, por diversas razones, a saber: marco conceptual interpretable, se expresa en el lenguaje propio del grupo participante, genera un resultado gráfico o repre-
sentativo que simultáneamente muestra todas las ideas principales y las relaciones entre ellas; a menudo fortalece grupos, de los participantes en el grupo de trabajo.

Probablemente el primer paso es el más difícil en un proyecto de planificación o evaluación, todo lo que sigue depende de lo bien que se haya concebido inicialmente el proyecto. En este
sentido, la acción de conceptualizar se refiere a la articulación de pensamientos, ideas o intuiciones y su representación mente se desean definir las metas y los objetivos principales, que pueda formar parte, eventualmente, de los elementos del plan. En la evaluación, se desean conceptualizar los prograque se consideren relevantes.

Para construir el mapa, una vez concebidas las ideas deben ser descritas, y la interrelación entre ellas articulada. Tecnicas esde correspondencias y análisis de grupo - son luego analisis y los resultados representados en un mapa, en planos con eje de abscisas y gráficos. El contenido del mapa es totalmente determinado por el grupo. Los participantes se someten a un fase llamada tormenta de ideas en la cual crean sus primeros enunciados, proveen información acerca de cómo están interrelacionados ellos mismos, interpretan los resultados de los anál(a)

Se asume que en el escenario en el cual se aplica el diseño de para guiar el trabajo de evaluación. Dependiendo de la situación este grupo puede estar constituido por administradores, trabajadores o miembros de la junta directiva de una organización Ideres de la comunidad o representantes de grupos de la gerencia acticis o legisladores raría: representantes de grupos de usuarios de la de contración pública; o una combinación de todos estos. El proceso de diseño lo conduce un coordinador responsable de la evaluación o análisis, que bien puede ser parte o miembro de la organización donde se realiza el trabajo o puede ser mente en dirigir el proceso -el contenido, la insonsiste sola mente en dirigir el proceso -el contenido, la interpretación y
la utilización del mapa conceptual va a estar completamente determinada por el grupo-

Ahora bien, hay dos tareas fundamentales que deben ser llevadas a cabo antes de comenzar el proceso en grupo. En primer lugar, el coordinador debe decidir quienes van a participar en to con los participantes 0 con un subgrupo para deciar junva a ser el enfoque espećfíco de la herramienta de trabajo. selección de colaboradores, es la decisión de quien participarà en la sesión de grupo y el diseño del mapa conceptual. Es una de las tareas más importantes del coordinador. La experiencia demuestra que un buen diseño incluye una amplia variedad de personas. En un contexto de programa de evaluación, se podria ma usuarios, teóricos de las ciencias sociales, miembros de nidad y miembros de fundaciones relacionadas al área.

En este estudio, el propósito del trabajo fue reunir un pequeño conjunto de representantes de distintas instituciones que se elacionan con el estudio de la seguridad social, las pensiones y jubliaciones en Venezuela para emerger variables claves para proceso de diseño del mapa conceptual con la idea de que posiblemente pudiera ser aplicado más tarde, en la generación de futuras líneas de investigación, con un grupo más amplio en el que se incluyeran más personas. Pues bien, con base a técnica de Trochim (1989), seleccionaron entre cinco (5) y diez (10) personas participaron en las dos reuniones que se relacionadas con el área de la gerencia de recursos humanos, administración de justicia (jueces), profesores universitarios e investigadores, pensionados y jubilados. Fortaleciendo la con el tema investigado.

El segundo paso está relacionado con el desarrollo del enfoque o focalización del trabajo. En el proceso del conversatorio del Sistema de Seguridad Social, pensiones y jubilaciones se
había hecho llegar a los invitados previamente un resumen con había hecho llegar a los invitados previamente un resumen con la metodología a seguir y el enfoque del tema. Con esto se
buscaban dos resultados: Primero, los participantes definen la buscaban dos resultados: Primero, los participantes definen la los participantes precisan el enfoque para jerarquizar en orden los enunciados lo cual es una etapa que tiene lugar en el paso de estructuración. Esto esencialmente quiere decir que se tiene que definir la posición donde va a ser ubicado cada enuncia (variable) originado en la sesión de tormenta de ideas.

ideas fue bastante amplia ionfocíndonosión de tormenta de ideas fue bastante amplia enfocándonos en la composición
de frases que describieran temas de interés en la reforma e mplementación de la Ley Orgánica de Seguridad Social, o simplemente como ciudadano beneficiario de la ley, exponer la perspectiva, preocupación, experiencia o hallazgo que sume valor agregado a la investigación. Aunque esto pueda parecer

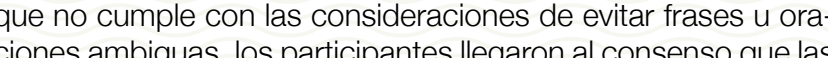
ideas generadas luego de la discusión constituirían un conjunto descifrable y relativamente homogéneo.

Una vez que los participantes y los enfoques han sido definidos, comienza el verdadero proceso de diseño con la generación de un conjunto de enunciados que inmejorablemente representarán mente, el coordinador registra todas las ideas a medida que son generadas de manera que todos los miembros del grupo puedan ver el conjunto a medida que se va desarrollando. Esto se puede hacer simplemente escribiendo los enunciados en un pizarrón o en hojas de papel. En este caso, los enunciados fue mostraron en una pantalla grande de manera que todos los participantes los pudieran ver. La sesión del conversatorio fue grabada en video en su totalidad para no perder ningún detalle de la discusión y de la generación de los resultados.

También, se tomó la previsión para evitar que algunos paricipantes no estuviesen dispuestos a exponer publicamente da, entonces se le permitió a cada uno de ellos presentar los enunciados/ideas de manera anónima en papel, con el fin de mantener la confidencialidad. Teóricamente, no hay límite para el número de enunciados que pueden ser concebidos. Sin embargo, los números grandes imponen algunas restricciones prácticas.

Una vez que el conjunto definitivo de enunciados se ha concretado, el grupo revisa la redacción. Algunas veces la transcripideas no es la más adecuada, o en ocasiones la jerga técnica no es clara. Por lo general, todos los enunciados deben concordar con lo que se pidió originalmente y deben presentarse claramente, de manera que todos los participantes del grupo puedan entender es sentido esencili dos mismos.
La prospectiva y el análisis estructura

Antes de seguir exponiendo acerca de las fases del análisis esthe Generamente, este tipo de técnicas apunica la prospecpara visualizar en este tipo de tecnicas apuntan a un análisis en el futuro. Por ello, el aporte de la teoría prospectiva es la configuración de un grupo que senala mediante una serie de firmaciones una problemática desde un contexto que ha sido diagnosticado previamente.

En este sentido, la prospectiva, es un método a través del cual ersigue primero determinar el futuro deseado para contrasque conducirá hacia ese futuro deseado. Se concreta como una disciplina que facilita el análisis de situaciones con el fin e aprovechar las oportunidades internas y externas, para adelantarse a las tendencias y a través de la investigación facilitar a stores sociales la construcción de un futuro" (Ministerio de

Este trabajo se toman la incidencia de ciertas variables que filuyen en el desarrollo de una politica social, y vista la metodología que se ha desarrollado, Godet (1999), explica la importancia na lista lo más completa posible de las variables a tener en cuenta, cuantificables o no, con el fin de tener una visión global tan exhaustiva como sea posible del sistema que constituye el de este estudio la lista de variables a tener en cuenta estaría configurada tal como se muestra en la Tabla No. 2

Las variables, en el presente estudio surgieron de las declaracones expresadas por los expertos en el conversatorio soquienes se reseñan en la Tabla No. 1, constituyendo un punto de partida fructifero para el análisis. Estas variables se centran en la búsqueda de los determinantes principales del sistema y de sus parámetros más sensibles, pasa por el examen de los efectos directos e indirectos del entorno general (variables ex(as) sobre las variables que caracterizan el fenómeno.

Tabla 1. Perfil de los participantes en los Conversatorios

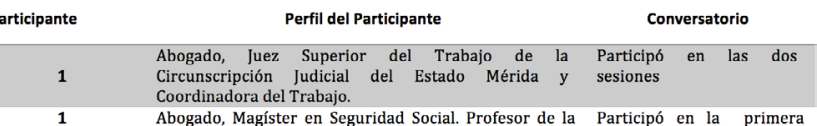

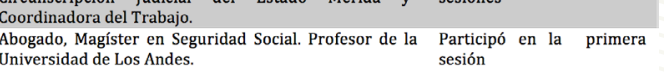

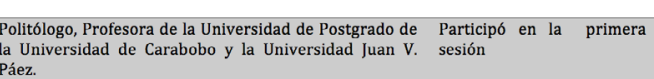

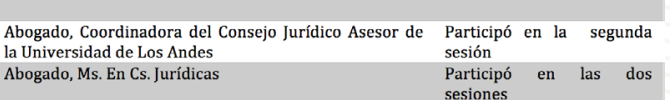

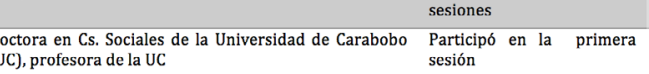

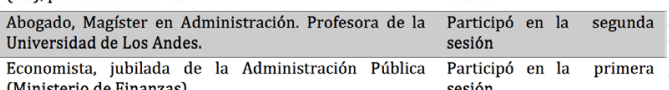

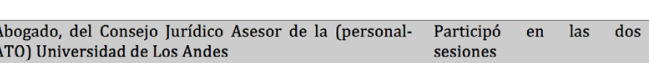

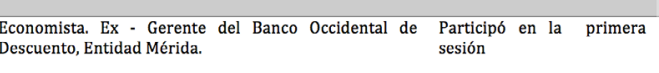
Licencia
Andes. 
Tabla 2. Resultado del Conversatotorio: Variables más destacables y su definición

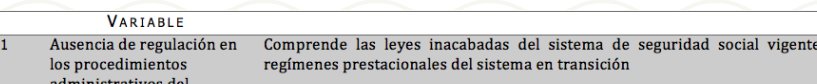

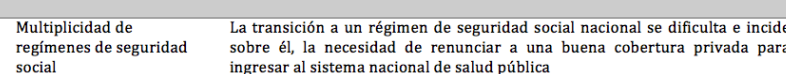

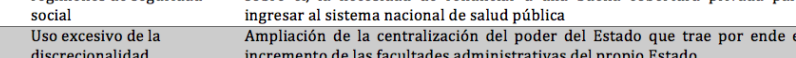

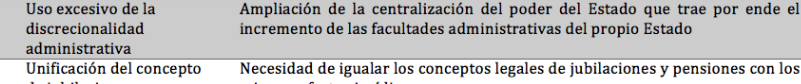

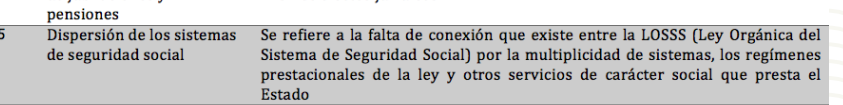

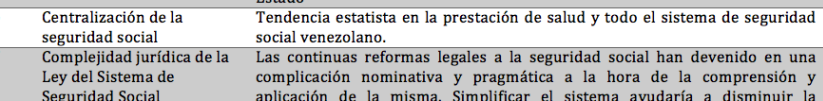

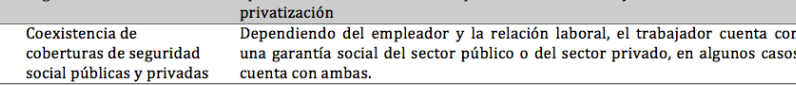

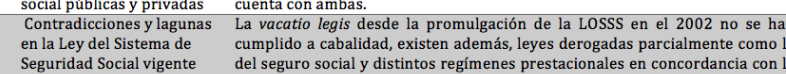

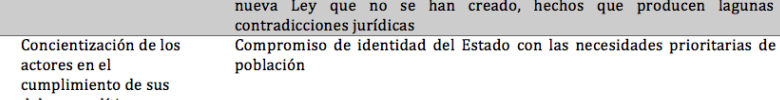

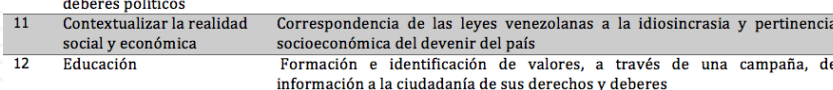

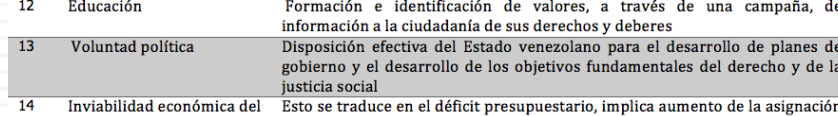

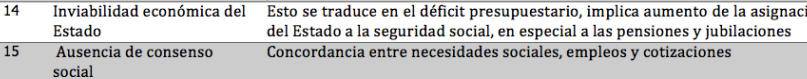

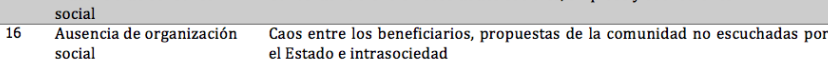

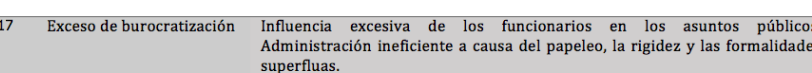
18 Factor demografficico

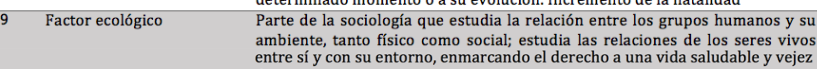

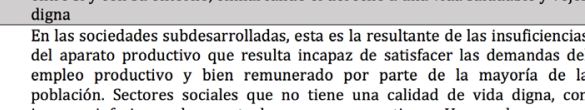

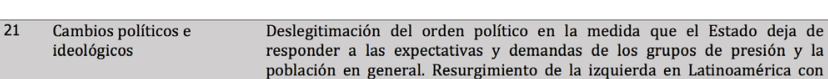

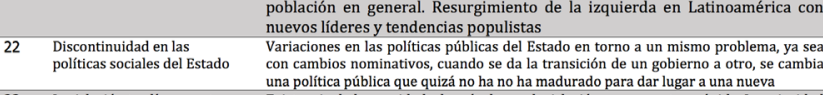

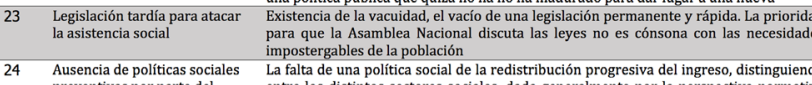

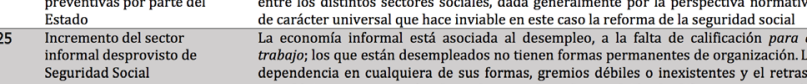
Fuente: Elaboración propia (2017) Ahora bien, en este punto cuando se tiene el conjunto de enunciados que describen el dominio conceptual, es imperativo examinar requiere que cada enunciado sea colocadodo en un rango que este definido por lo expuesto en el enfoque y en orden jerárquico. Estas dos tareas constituyen lo que se llama la estructuración del dominio
conceptual. Esto se consiguió desarrollando una Matriz de Impacto Cruzado (MICMAC)

Una vez que el grupo en el conversatorio termina con el proceso de puntuación se procede a hacer el vaciado de la información en este Investigaciones Prospectivas Estratégica y Organización (EI Lipsor, 2001); este permite generar gráficos mostrado como influyen variables entre sí, como se puede observar en la Figura No. 1

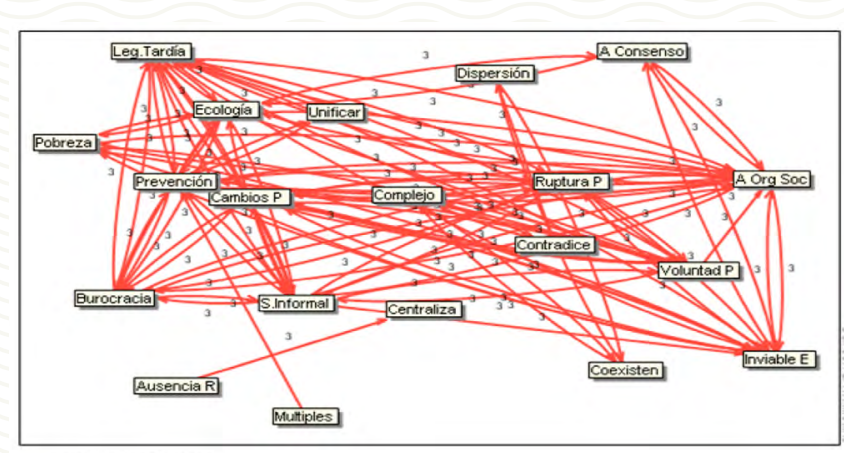

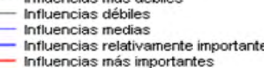

Figura 1. Variables con influencias directas en el sistema de Seguridad Social En este gráfico se puede ver que las variables del sistema de Seguridad social, están fuertemente interrelacionadas, lo que significa que cualquier influencia ejercida sobre una de ellas afecta a todas las demás y viceversa, pero, además, esto indica que son, por tanto, bastante homogeneas. Esta homogeneidad depende de la función que estas variables pueden

De esta manera, se interpreta que todas las variables de cada componente del sistema que inciden sobre el sistema de seguridad social están estrechamente relacionadas entre sí, por tal razón, se denominan como macro variables. Estas macro variables incluyen variables que están, a su vez, íntimamente relacionadas y que son, en consecuencia, también funcionalcos, es que el gráfico simplificado que se logra después de integrar las macro variables también se puede organizar en un árbol jerárquico.

En resumen, es conveniente entender la preponderancia asistencia de las variables identificadas en los bucles de influencia, para retomar la implementación de la seguridad social y las pensiones en Venezuela de una manera expedita, ya que las por su inmanente condición social. tiva, es que a la larga mejore la calidad de vida del venezolanto. Estando el quid en la implementación de políticas que desvien el peso del financiamiento del sistema de pensiones sólo en e Estado, dejando entrever la mixtura del sistema (Rivas T. Frank E. y Villasmil María A., 2015)

\section{Reflexiones}

, las ventajas del método se encuentran en que y hace cavilar sobre los aspectos contra-intuitivos del grupo portamiento de un sistema. Está claro que no hay una lectura única y oficial de resultados del MICMAC y conviene que grupo haga avanzar la reflexión con nuevas interpretaciones. Por otra parte, el metodo presenta la ventaja de permitir un estudio cualitativo del sistema en el cual se sustenta la seguridad social venezolana y el Régimen Prestacional de Pensiones Jubilaciones. investigaciones realizada un sistema de pensiones, representa más que un texto juríí$c o$, representa la encarnación de mútiples cambios, reformas y contrarreformas que intenten proteger al ciudadano, tal parece que la tarea que que in el Es polo ver económica, incremento de la burocracia, los cambios pol'ticicos e ideológicos, la complejidad legislativa y hasta la corrupción que han conspirado negativamente para alcanzar el éxito en la meta propuesta.

Por último, cabe mencionar que los límites del método se relacionan, en primer término, con el carácter subjetivo de la lista de variables elaboradas durante la primera fase, e igualmente con relación con los actores del sistema. El juicio personal suele ser el único elemento de información accesible para tomar en cuenta los acontecimientos que podrían producirse. No hay esadísticas de futuro, expresa el Laboratorio de Investigaciones Prospectivas -LIPSOR- (2001). De otra parte, la matriz contiene las relaciones de intensidad muy direrentes que deberán tenerse en cuenta entonces para su tratamiento. En fin, hay que conceder la sensibilidad de los resultados a una variación dos al pie de la letra, sino que su finalidad, como ya se dijo, es solamente suscitar la reflexión. "Boletín de Coyuntura" y "Revista de Coyuntura" OBEST

Callejo, (2001), El grupo de discusión de introducción a una
práctica de investigacion. EDITORIAL ARIILL. Barcelona, ESpaña. EI Lipsor (2001). Cf. M. Godet, Manuel de prospective
stratégique, fome 2 Editions Dunod. Cf. M. Godet, Creating Futures

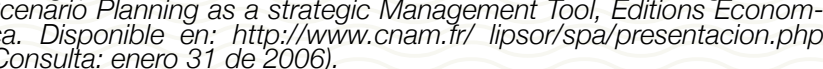
Godet, Michel (1999). De la anticipación a la acción. Manual de
prospectiva y estrategia. Afta omega, Santa Fe de Bogotá, Colombia.
p. 26.

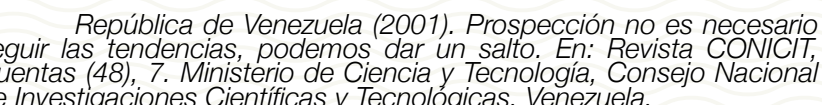

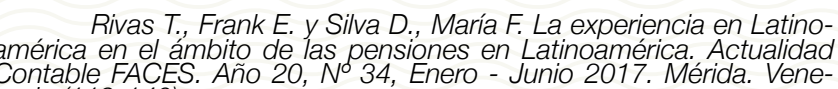

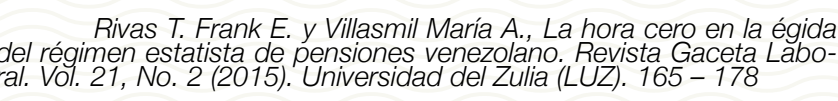
Trochim, Guillermo (1989). Una introducción para el diseño de
conceeptualus utilizados en la planificación y evaluación. UniverTrochim, W. \& Linton, R. (1989). Conceptualization for evalua-
ion and planning. Evaluation and program planning. . Universidad de

\section{Guía para presentación de artículos}

Originalidad
Los aticulos
ninguin medio

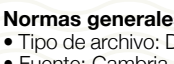

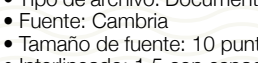

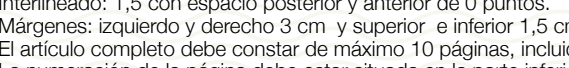

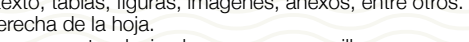

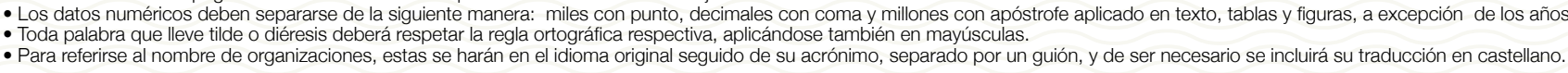

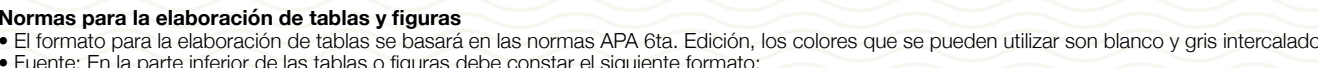

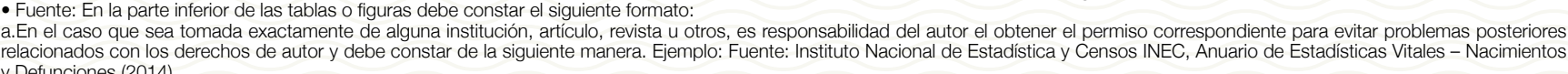

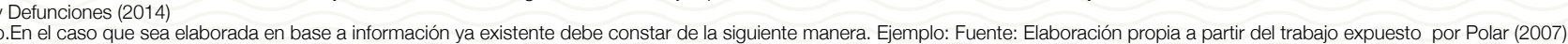

Estructura del articulo

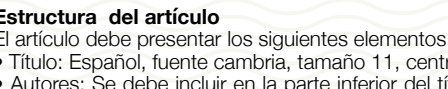

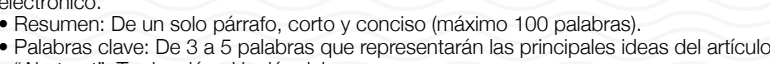

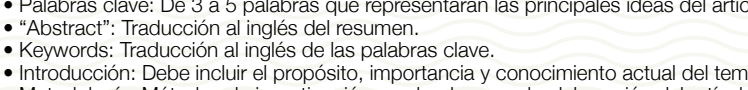

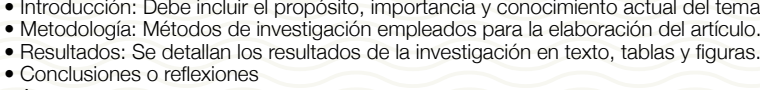

- Anexos Reterencias: Normas APA Gta edición en orden altabetico y sangria francesa.

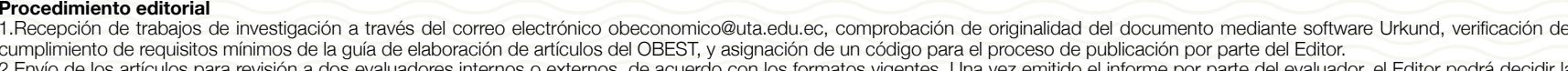

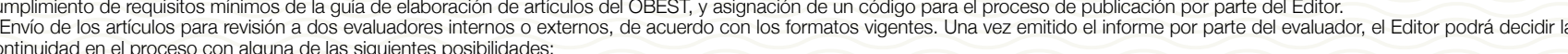

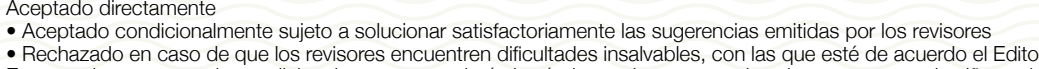

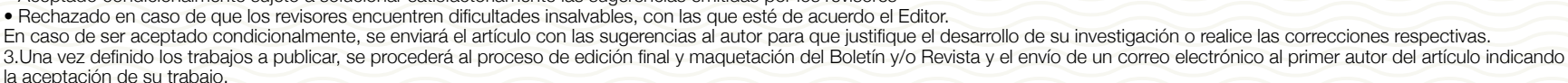

